\title{
A rare case of lymphangiomatosis
}

D Parris, ${ }^{1}$ BSc, MB BCh, FCPaed, Dip Allergy; A van Niekerk, ${ }^{1} \mathrm{MB}$ ChB, MMed; E Marais, ${ }^{2} \mathrm{MB}$ ChB, MMed Anat Path;

I O Lawal, ${ }^{3}$ MBBS; A Goga, ${ }^{1} \mathrm{MB}$ ChB, DTM\&H, DCh, MSc, MCh, MSc Epidemiology, FCPaed, PhD;

A C Jeevarathnum, ${ }^{1} \mathrm{MB}$ BCh, FCPaed, Dip Allergy, MMed, Cert Paed Pulm, European Respiratory Diploma;

X Dearden, ${ }^{1} \mathrm{MB}$ ChB, FCPaed, MMed, Dip Allergy; R J Green, ${ }^{1} \mathrm{PhD}$, DSc

\author{
${ }^{1}$ Department of Paediatrics and Child Health, School of Medicine, Faculty of Health Sciences, University of Pretoria, and Steve Biko Academic Hospital, Pretoria, \\ South Africa \\ ${ }^{2}$ Department of Anatomical Pathology, School of Medicine, Faculty of Health Sciences, University of Pretoria, and Steve Biko Academic Hospital, Pretoria, \\ South Africa \\ ${ }^{3}$ Department of Radiology, School of Medicine, Faculty of Health Sciences, University of Pretoria and Steve Biko Academic Hospital, Pretoria, South Africa
}

Corresponding author: D Parris (drdeniseparris@gmail.com)

Lymphangiomatosis is a rare disorder and the underlying aetiology is poorly understood. The diagnosis is difficult, and relies on various clinical, radiological and histological features. Therapy is diverse, with combinations of treatment required to achieve disease control. The prognosis is guarded, and currently a vast amount of work is being undertaken to understand the disease, and to find focused therapy. We present a case of histologically, and radiologically proven lymphangiomatosis in a 4-year-old child who presented with nonspecific symptoms.

S Afr Respir J 2017;23(2):35-38. DOI:10.7196/SARJ.2017.v23i2.153

Lymphangiomatosis is thought to be an intrauterine anomaly that occurs during embryogenesis, with unchecked proliferation of lymphatic vessels. ${ }^{[1,2]}$ Currently, no genetic, immunological or environmental factors have been identified in the pathogenesis of the disease. The clinical manifestations are diverse, and can occur as an isolated disorder, like a cystic hygroma, or can present with profound cardiovascular involvement, chylothoraces, and chylous pericardial effusions. ${ }^{[3]}$ The diagnosis is made on a biopsy of the involved site, and demonstration of dilated lymphatic channels, with positive staining for D2-40 and CD-31 antigens. ${ }^{[4]}$ Since the pathophysiology of the disease is poorly understood, therapy is largely experimental, with variable outcomes. ${ }^{[1,2,4,5]}$

\section{Case report}

A 4-year-old female child was referred with a left-sided chest mass, extending from the 3 rd to the 6 th rib laterally, and extending anteriorly over the mid-clavicular line. It was soft, non-tender, not pulsating, and $10 \mathrm{~cm} \times 15 \mathrm{~cm}$ in size. She had developed a chronic cough, which was initially dry, but had become progressively more productive. There was no associated significant history.

Clinically, the child was well-grown, with no features of clubbing or anaemia. The mass was palpable over the left chest wall. The percussion note was stony-dull and breath sounds were markedly decreased over the left chest, in keeping with a pleural effusion. The rest of the examination was unremarkable.

Her laboratory evaluation revealed a mild anaemia. The HIV ELISA test result was negative. Both the Mantoux test, and the Gene-Xpert results for pulmonary tuberculosis were negative. There was no evidence of infection on blood screening. Erythrocyte sedimentation rate, renal functions, liver function tests, and thyroid function tests were all within normal limits.
Her pleural tap, under ultrasound guidance, was diagnostic for a chylothorax - both clinically, and on biochemical analysis of the pleural fluid (Table 1).

\begin{tabular}{ll}
\multicolumn{2}{l}{ Table 1. Biochemical analysis of the pleural fluid } \\
\hline Parameter & Value \\
\hline $\mathrm{pH}$ & 7.6 \\
Colour & Milky \\
Total fat & $5 \mathrm{~g} / \mathrm{dL}$ (elevated) \\
Cholesterol & $1.8 \mathrm{mmol} / \mathrm{L}$ (elevated) \\
Triglycerides & $1.9 \mathrm{mmol} / \mathrm{L}$ (elevated) \\
Total protein & $4 \mathrm{~g} / \mathrm{dL}$ \\
Electrolytes & Equivalent to plasma \\
Glucose & $4.8 \mathrm{mmol} / \mathrm{L}$ \\
Chylomicrons & Positive
\end{tabular}

Chest X-ray (CXR) revealed a massive left-sided effusion (Fig. 1) The cardiac assessment was normal, with no evidence of pulmonary hypertension or cor pulmonale. Her computed tomography (CT) scan (Figs 2A - 2D) demonstrated a left-sided effusion, with extensive destruction of the left lung. Multiple lytic lesions were visible in the spleen, and the vertebrae of T3 and T4. Lymphoscintigraphy of the chest demonstrated evidence of a lymphatic leak, but due to the extensive lymphatic involvement, the exact site was not delineated.

Owing to the progressive deterioration of the patient's clinical condition, a pleural biopsy was performed under general anaesthesia, and this result was diagnostic. The biopsy findings were pathognomonic of diffuse pulmonary lymphangiomatosis (DPL). Variable amounts 


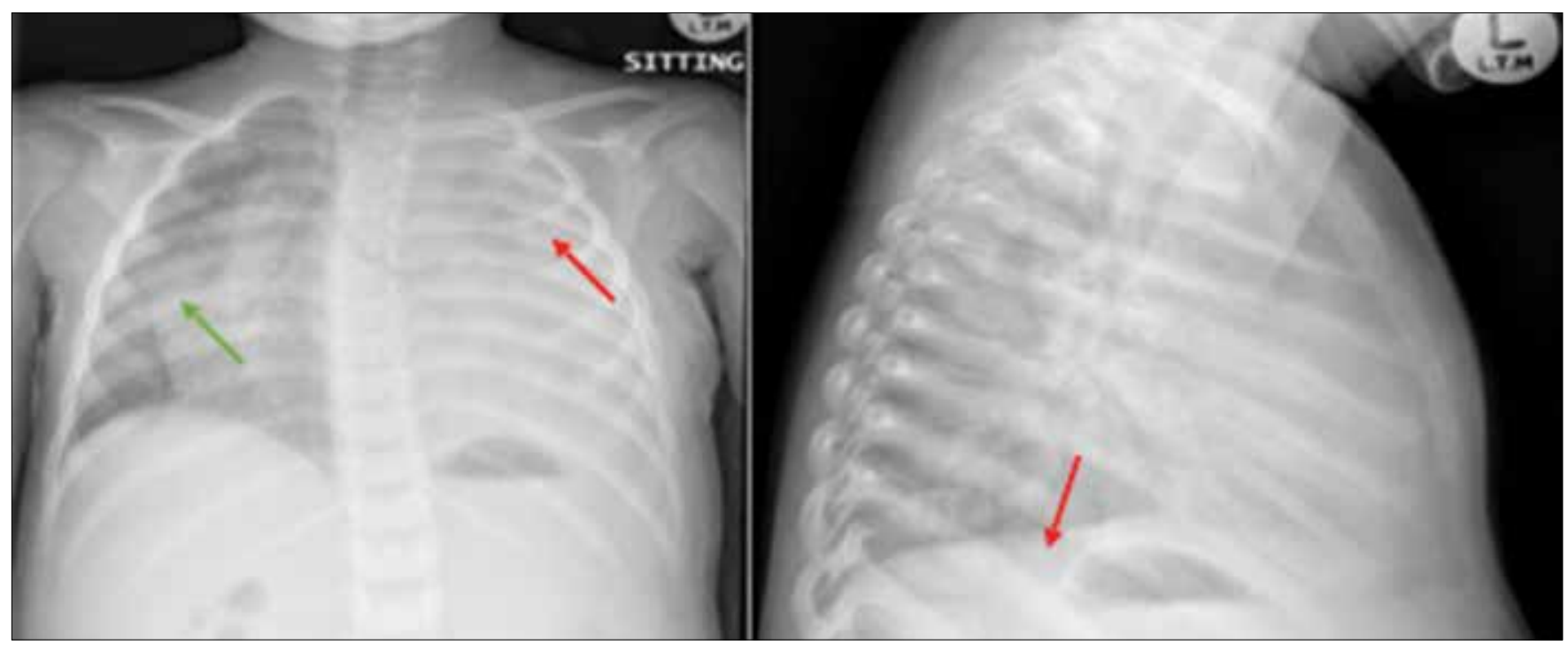

Fig. 1. Massive left-sided effusion (green arrow), with mediastinal shift (red arrows).
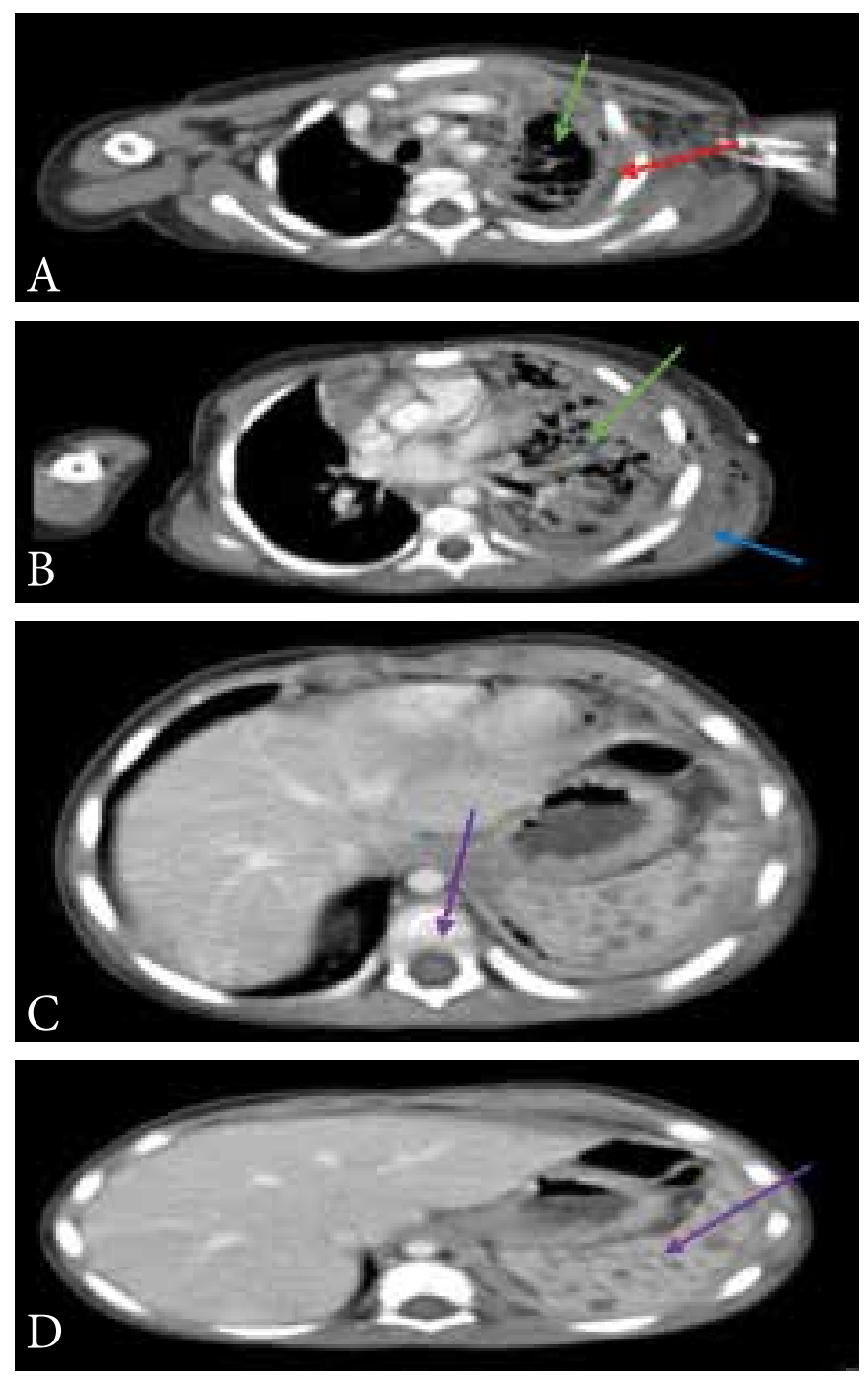

Fig. 2. Contrast scan of the lungs, vertebrae, and abdomen. (A) Extensive pleural effusion (red arrow) with lung consolidation, and cystic changes (green arrow). (B) Effusion with consolidation, trapped air (green arrow), and a soft tissue mass on the left (blue arrow). (C) Lytic lesions in the vertebrae (T3 and T4). (D) Extensive lytic lesions present in the spleen (purple arrow). of fibrosis, with areas of focal haemorrhage, were demonstrated. Aggregates of inflammatory cells, mainly small lymphocytes, were visualised. Diffuse and complex dilatation, with irregularity of lymphatic vessels, and lined by a single layer of endothelial cells, was recorded. The anastomosing spaces were filled with eosinophilic material and chyle. The cells stained positively for D2-40, and CD31 antigen (Figs 3A - 3D). This confirmed the diagnosis of DPL.

Treatment with dietary modification, along with glucocorticoids, and propranolol, was implemented. The propranolol treatment was implemented at a starting dose of $0.5 \mathrm{mg} / \mathrm{kg}$, and increased gradually, but was stopped owing to severe diarrhoea, vomiting, headache, and bradycardia. Propanolol treatment was stopped, but restarted a week later at $0.5 \mathrm{mg} / \mathrm{kg}$. The drug was slowly increased to $1 \mathrm{mg} / \mathrm{kg}$ over the next 2 weeks, and was better tolerated. The effusion reaccumulated, despite the above therapy and repeat pleural drainage. A PleurX catheter was inserted, and the mother received extensive training with respect to the management of the catheter. At the time of publication of this manuscript, the patient's diet was monitored by a dietician and she was she was being followed up at our clinic.

\section{Discussion}

Lymphangiomatosis is a rare disorder. The underlying aetiology is poorly understood, but recent research suggests that vascular endothelial growth factor receptor-3 (VEGFR-3) may be involved in the development of lymphangiomatosis. ${ }^{[1]}$ The process is thought to occur during embryonic growth. ${ }^{[1,6]}$ There are currently no genetic, immunological, or environmental factors implicated in the causation of the disorder. The malformations may occur in utero as part of another syndrome. Syndromes commonly associated with lymphatic malformations include Noonan syndrome, Turner syndrome, and Down syndrome. ${ }^{[1,2]}$ The disorder is characterised by masses of fluidfilled channels that can occur anywhere in the body, and may be either focal or diffuse. ${ }^{[7]}$

The lymphatic malformations can present at any age. Typically, congenital malformations present as soft, spongy, non-tender masses. ${ }^{[1,8]}$ In older children, the presentation is dependent on the site of the lesion, and the mass effect on adjacent structures. Infection, trauma, or bleeding into the malformation may result in rapid expansion of the lesion. 

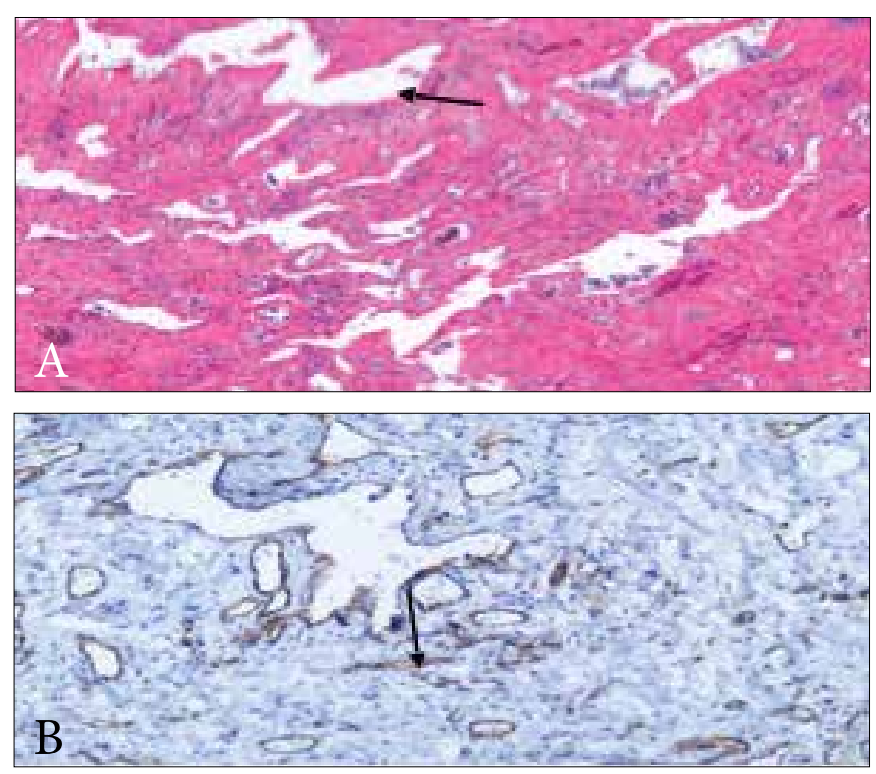

Fig. 3. Immunochemical staining of the lung and pleural biopsy specimen. (A) Histologically, the lung biopsy revealed an increase in the size and number of thin-walled channels lined by attenuated endothelial cells $(H \& E \times 40)$. (B) The lung biopsy revealed an increase in the size and number of thin-walled channels lined by attenuated endothelial cells with lymphocytes visualised $(H \mho E \times 100)$. (C) Immunohistochemical staining $(\times 200)$ with D2-40 antigen showing proliferation of the lymphatic channels, and strongly highlights the lining of the channels. (D) The attenuated endothelial cells lining the anastamosing irregular lymphatic channels where strongly positive for anti-CD31, which is highly specific for vascular endothelium (arrow) (Immunoperoxidase and anti-CD31 × 200).

Clinically, patients may present with disfiguring lesions over the body or upper airway obstruction from lesions, involving the mouth, tongue, and trachea. Swallowing and speech may be affected with supraglottic lesions. ${ }^{[9]}$ Lung lesions can cause marked airway compromise. Gastrointestinal involvement may result in severe weight loss, while splenic involvement causes severe abdominal pain. Bone involvement can lead to either bone overgrowth or bone loss, with an increased risk of pathological fractures. This disease entity is known as Gorham-Stout syndrome, which is also referred to as vanishing bone disease. ${ }^{[10,11]}$

Pulmonary lymphangiomatosis can have a varying clinical presentation, from being asymptomatic to profound respiratory failure. ${ }^{[1,2,7,12]}$ The disease is more rapidly progressive in children, where the clinical features reported include chronic cough, recurrent respiratory tract infections, haemoptysis from vessel erosion, and chyloptysis. ${ }^{[1,3,12]}$ Dyspnoea, chest pain, and chylous pleural effusions, are other associated features. The chylous effusions can be large, causing airway compromise. ${ }^{[13]}$ Complications associated with DPL include compression of the adjacent chest wall, with lytic lesions in the bone. ${ }^{[13]}$ Pneumothoraces have also been reported. Chylous pericardial effusions may produce cardiac tamponade, with hypotension. ${ }^{[5,12]}$ Pulmonary hypertension and cor pulmonale are features of this disease. The chylous pleural effusions are a nidus for infection, with severe pneumonias occurring in already compromised respiratory patients. Disseminated vascular coagulation is a rare complication. ${ }^{[8,12]}$

Chest radiographs are not diagnostic in DPL, but may reveal diffuse interstitial patterns, and pleural effusions. Contrasted computed tomography (CT) scans demonstrate bilateral, smooth thickening of the interlobular septae and bronchovascular bundles, with patchy ground-glass opacities, as well as diffuse fluid accumulation in the mediastinum and hilar soft tissue. Unilateral or bilateral pleural effusions, with pleural thickening can also be observed. Not all the features may be present in a patient. These findings are not conclusive for DPL, but may allude to the possibility of the disease. ${ }^{[2,9,12,14]}$ Lymphoscintigraphy or lymphangiography can be useful in outlining the lymphatic vessels, and in identifying the site of the lymphatic leak. These investigations both require administration of a contrast media. A simultaneous CXR, or CT scan, will outline the lymphatic anatomy. The sensitivity of this investigation is low in DPL..$^{[6,8,15]}$ Bronchoscopy is of limited value, and most diagnoses of DPL are based on open lung biopsy followed by immunohistochemical staining. ${ }^{[4]}$ Pulmonary function tests were shown to reveal both restrictive and obstructive patterns. ${ }^{[3,7]}$ This investigation is difficult in the young child.

Treatment options are varied, and no single therapy has proven to be successful. For localised disease, surgical resection may be therapeutic. ${ }^{[9]}$ For DPL, dietary modification with a low-fat diet, and medium-chain triglycerides, along with albumin infusions, has demonstrated little success. ${ }^{[12]}$ Sclerotherapy using doxycyline as the sclerosing agent has resulted in improvement in some patients; however, this form of therapy requires multiple injections of doxycycline, and is of limited value in extensive disease. ${ }^{[16]}$ Two commonly employed therapies include interferon alfa-2b (INF-2b) and glucocorticoids. Successful treatment was achieved in a 5 -year-old boy who presented with a right-sided pleural effusion. Thoracocentesis revealed a chylous effusion, and a chylothorax was confirmed on immunohistochemical analysis that demonstrated the positive CD31 and CD34 markers for vascular endothelial cells; some of the endothelia stained positive for the monoclonal antibody, D2-40. This is a confirmatory test for lymphangiomatosis. Wholebody MRI on the boy confirmed disseminated disease. PEGylated INF-2b, was administered weekly at a dose of $1.0 \mu \mathrm{g} / \mathrm{kg}$. After the second dose of INF-2b, $1.8 \mathrm{~L}$ of chylous fluid was evacuated from the pleural space. The third dose of the drug was increased to $1.5 \mu \mathrm{g} / \mathrm{kg}$. The child's clinical condition improved, and after 3 months of therapy, his lung function, exercise tolerance, chest radiographs, and bone lesions showed marked improvement. He experienced some minor side-effects, including headache, raised temperatures, and malaise. These side-effects were transient. He was on INF therapy for 9 months with no further disease progression, and improved quality of life. ${ }^{[1,17)}$ Other drugs used include biphosphonates, thalidomide, and rapamycin. ${ }^{[1]}$ Octreotide is a synthetic analogue of somatostatin, which is an endogenous hormone, has been used with some success in the reduction of chyle production within the lymphatic system; however, the side-effect profile has limited its clinical use. ${ }^{[18]}$ More recently, propranolol and bevacizumab have been found to inhibit synthesis of vascular endothelial growth factor. ${ }^{[4,19,20)}$ Both these drugs have demonstrated encouraging results. Propranolol was used in a 13-year-old boy, in whom the dosage of PEGylated INF$2 \mathrm{~b}$ was decreased as a consequence of marked side-effects, including depression. The effusion reaccumulated despite increasing the dose of PEGylated INF-2b. The volume of chylous fluid drained daily was $>100 \mathrm{~mL}$. Treatment with propranolol was commenced at $0.5 \mathrm{mg} / \mathrm{kg} /$ day, in three divided doses. The daily volume of chylous fluid declined slowly, allowing the INF-2b to be discontinued. He 
received 10 months of daily propranolol, with improved quality of life, and decreased drainage of pleural fluid. ${ }^{[1,19]}$ Bevacizumab was successfully used on a 40-year-old female, in whom the diagnosis of pulmonary lymphangiomatosis was delayed for 3 years. She presented with haemoptysis initially, and subsequent mediastinal masses, pleural masses, and splenic disease. Revision of her previous histology confirmed diffuse pulmonary lymphangiomatosis. Intravenous bevacizumab, at a dose of $1 \mathrm{mg} / \mathrm{kg}$, was administered intravenously every 3 weeks. The haemoptysis resolved, the blood haemoglobin levels stabilised, and the masses decreased in size; however, therapy was aborted after seven treatments, as the patient developed hypertension. The patient did not have further episodes of haemoptysis and, 10 months post cessation of bevacizumab, the masses had not increased in size. ${ }^{[1,20]}$ Radiotherapy has been used for DPL where surgery is contraindicated, with poor results and the potential for lung fibrosis. ${ }^{[1]}$ Only one case of successful lung transplantation has been reported. ${ }^{[15]}$

Acknowledgements: Prof. R. Green for being an amazing project supervisor.

Author contributions: DP wrote the manuscript. EM (Department of Histopathology) prepared the slides. IOL (Department of Radiology) analysed the scans. AVN reviewed the patient with DP. RJG reviewed the manuscript.

Funding: None

Conflicts of interest: None

1. Tazelaar HD, Kerr D, Yousem SA, Saldana MJ, Longston C, Colby T. Diffuse pulmonary lymphangiomatosis. Human Pathol 1993;24(12):1313-1322. https://doi. org/10.1016/0046-8177(93)90265-i

2. Faul JL, Berry GJ, Colby TV, et al. Thoracic lymphangiomas, lymphangiectasis, lymphangiomatosis, and lymphatic dysplasia syndrome. Am J Respir Crit Care Med 2000;161(3):1037-1046. https://doi.org/10.1164/ajrccm.161.3.9904056

3. Satria MN, Pacheco-Rodriguez G, Moss J. Pulmonary lymphangiomatosis. Lymphat Res Biol 2011;9(4):191-193. https://doi.org/10.1089/lrb.2011.0023

4. Ramani P, Shah A. Lymphangiomatosis: Histologic and immunohistochemical analysis of four cases. Am J Surg Path 1993;17(4):329-335. https://doi.org/10.1097/00000478199304000-00002
5. Nakagawa T, Koizumi T, Oiwa K, et al. Sudden death of a 14-year-old girl with lymphangiomatosis. Gen Thorac Cardiovasc Surg 2016;64(2):116-119. https://doi. org/10.1007/s11748-014-0450-6

6. Bellini C, Villa G, Sambucetti G, et al. Lymphoscintigraphy patterns in newborns and children with congenital lymphatic dysplasia. Lymph 2014;47(1):28-39.

7. Zhao J, Wu R, Gu Y. Pathology analysis of a rare case of diffuse pulmonary lymhangiomatosis. Ann Transl Med 2016;4(6):114. https://doi.org/10.21037/ atm.2016.03.30

8. Fukahori S, Tsuru T, Asagiri K, et al. Thoracic lymphangiomatosis with massive chylothorax after tumor biopsy and with disseminated intravenous coagulation lymphoscintigraphy, an alternative minimally invasive imaging technique: Report of a case. Surg Today 2011;41(7):978-982. https://doi.org/10.1007/s00595-010-4383-0

9. Perkins JA, Manning SC, Tempero RM, et al. Lymphatic malformations: Review of current treatment. Otolaryngol Head Neck Surg 2010;142(6):795-803. https://doi. org/10.1016/j.otohns.2010.02.026

10. Yavasoglu I, Cakiroglu U. Diffuse lymphangiomatosis: Gorham-Stout syndrome. Intern Med 2014:53(1):75-76. https://doi.org/10.2169/internalmedicine.53.0987

11. Gordon KD, Mortimer PS. Progressive lymphangiomatosis and Gorhams's disease: Case report and clinical implications. Lymphat Res Biol 2011;9(4):201-204. https:// doi.org/10.1089/lrb.2011.0021

12. Kadakia KC, Patel SM, Yi ES, Limper AH. Diffuse pulmonary lymphangiomatosis Can Respir J 2013;20(1):52-54. https://doi.org/10.1155/2013/971350

13. Canil K, Fitzgerald P, Lau G. Massive chylothorax associated with lymphangiomatosis of the bone. J Pediatr Surg 1994;29(9):1186-1188. https://doi.org/10.1016/00223468(94)90796-x

14. Du MH, Ye RJ, Sun KK, et al. Diffuse pulmonary lymphangiomatosis: A case report with literature review. Chin Med J 2011;124(5):797-800. https://doi.org/10.3760/cma .j.issn.0366-6999.2011.05.033

15. James D. Chylothorax in infants and children. Pediatrics 2014:133(4):722-733. https:// doi.org/10.1542/peds.2013-2072

16. Burrows PE, Mitri RK, Alomari A, et al. Percutaneous sclerotherapy of lymphatic malformations with doxycycline. Lymphat Res Biol 2008;6(3-4):209-216. https://doi. org/10.1089/lrb.2008.1004

17. Ozeki M. Funato M. Clinical improvement of diffuse lymphangiomatosis with pegylated interferon alfa-2B therapy: Case report and review of the literature. Pediatr Hematol Oncol 2007;24(7):513-524. https://doi.org/10.1080/08880010701533603

18. Roehr CC, Jung A, Proquitte H, et al. Somatostatin or octreotide as treatment options for chylothorax in young children: A systematic review. Intens Care Med 2006;32(5):650-657. https://doi.org/10.1007/s00134-006-0114-9

19. Ozeki M, Fukao T, Kondo N. Propranolol for intractable diffuse lymphangiomatosis. N Engl J Med 2011;364(14):1380-1382. https://doi.org/10.1056/nejmc1013217

20. Aman J, Thunnissen E, Paul MA, van Nieuw Amerongen GP, Vonk-Noordegraaf A. Successful treatment of diffuse pulmonary lymphangiomatosis with bevacizumab. Ann Intern Med 2012;156(11):839-840. https://doi.org/10.7326/0003-4819-156-11201206050-00016

21. Kinnier CV, Eu JP, Davis RD, et al. Successful bilateral lung transplantation for lymphangiomatosis. Am J Transplant 2008;8(9):1946-1950. https://doi.org/10.1111/ j.1600-6143.2008.02340.x 